\title{
Generative models of cortical oscillations: neurobiological implications of the Kuramoto model
}

\author{
Michael Breakspear ${ }^{1,2,3,4}$, Stewart Heitmann ${ }^{1,2}$ and Andreas Daffertshofer ${ }^{5}$ \\ 1 School of Psychiatry, University of New South Wales, Sydney, NSW, Australia \\ 2 The Black Dog Institute, Prince of Wales Hospital, Sydney, NSW, Australia \\ ${ }^{3}$ Queensland Institute of Medical Research, Brisbane, QLD, Australia \\ ${ }^{4}$ Royal Brisbane and Women's Hospital, Brisbane, QLD, Australia \\ ${ }^{5}$ Research Institute MOVE, VU University Amsterdam, Amsterdam, Netherlands
}

\section{Edited by:}

Kai J. Miller, University of Washington USA

\section{Reviewed by:}

Carson Chow, University of Pittsburgh, USA; National Institutes of Health, USA Ole Paulsen, University of Cambridge, UK; University of Oxford, UK

\section{*Correspondence:}

Michael Breakspear, 300 Herston Rd Herston, QLD, 4009, Australia. e-mail:mbreak@unsw.edu.au
Understanding the fundamental mechanisms governing fluctuating oscillations in large-scale cortical circuits is a crucial prelude to a proper knowledge of their role in both adaptive and pathological cortical processes. Neuroscience research in this area has much to gain from understanding the Kuramoto model, a mathematical model that speaks to the very nature of coupled oscillating processes, and which has elucidated the core mechanisms of a range of biological and physical phenomena. In this paper, we provide a brief introduction to the Kuramoto model in its original, rather abstract, form and then focus on modifications that increase its neurobiological plausibility by incorporating topological properties of local cortical connectivity. The extended model elicits elaborate spatial patterns of synchronous oscillations that exhibit persistent dynamical instabilities reminiscent of cortical activity. We review how the Kuramoto model may be recast from an ordinary differential equation to a population level description using the nonlinear Fokker-Planck equation. We argue that such formulations are able to provide a mechanistic and unifying explanation of oscillatory phenomena in the human cortex, such as fluctuating beta oscillations, and their relationship to basic computational processes including multistability, criticality, and information capacity.

Keywords: cortical oscillations, neural synchrony, Kuramoto model, Fokker-Planck equation

\section{INTRODUCTION}

Over the last few decades, extensive neurophysiological research has established the intimate association between adaptive perceptual and behavioral processes and fluctuating oscillatory activity in the cortex. This occurs across a range of spatial and temporal scales, from percept-related changes in gamma oscillations recorded invasively within neuronal microcircuits (e.g., Bressler and Freeman, 1980), to motor-related modulations in cortical beta oscillations observable in extracranial recordings (e.g., Boonstra et al., 2007; Houweling et al., 2010). Fluctuations in beta amplitude also appear in spontaneous cortical activity (Freyer et al., 2009) but are greatly muted in a number of pathological conditions such as Parkinson's disease (Eusebio and Brown, 2009). Whilst neurophysiological data attest to the role of high frequency oscillations, there is also tremendous interest in slow frequency (below $0.1 \mathrm{~Hz}$ ) activity in resting state networks, as evident in functional neuroimaging data (Biswal et al., 2005). Activity in this field has almost exclusively been devoted toward empirical research, although related advances in computational neuroscience can provide important insights into the fundamental mechanisms of oscillatory activity in neuronal systems. We believe that unraveling the laws governing fluctuations in large-scale cortical oscillations is a necessary precursor to understanding their role in adaptive and pathological cortical functions.

Computational studies adopt a variety of abstractions in order to deal with complex dynamical systems like the brain. Models hence range from relatively simple algebraic forms, through increasing complexity, to very detailed networks of multi-compartment neurons connected via specific synaptic maps. Whereas the latter, detailed models allow the study of precise mechanisms to explain specific empirical observations, the former, more abstracted approach seeks to elucidate fundamental mechanisms that may underpin a variety of apparently diverse neurophysiological phenomena. The cortex has a very detailed cytoarchitectural and physiological make-up and, clearly, this detail is crucial to its many specific functions. However, "physiologically precise" models can quickly become highly parameterized, making systematic explorations of their dynamics an increasing challenge. Moreover, as we will show, even very simple dynamical systems are capable of both extraordinary spatiotemporal complexity and quite specific dynamics.

The present study is squarely positioned towards the more abstract, fundamental mechanisms end of the spectrum. In fact, in terms of oscillatory behavior - the focus of the present Special Issue - we study the most pared-back model achievable, the socalled Kuramoto model of coupled phase oscillators (Kuramoto, 1984). This model posits that the activity of a local system (neuron/ neural column/cortical area) can be sufficiently represented by its circular phase alone. Interactions amongst these entities, which collectively constitute a dynamical structure at the next coarsest spatial scale, are then introduced by a simple algebraic form that captures the essential characteristics of their exchanges, such as a post-synaptic transmembrane perturbation. In its simplest version, the Kuramoto model is a highly symmetrical and idealized system 
that can nonetheless exhibit rather non-trivial collective dynamics. Subsequently we introduce, and provide heuristic explanations for, a succession of increasingly less restrictive generalizations that boost the model's biological salience. Our objective is to communicate the essence of these adaptations, together with specific types of spatiotemporal complexity that they engender, e.g., synchrony, traveling waves, and dynamic instabilities. We aim to provide a neurobiologically-minded tour of the field, with relevant heuristic discussions of modifications of the Kuramoto model and some numerical illustrations of its wonderful dynamics ${ }^{1}$.

The paper is structured as follows. In the next section, the basic tenants of the Kuramoto model are introduced following Strogatz's (2000) erudite overview of the Kuramoto model (see also Acebrón et al., 2005). We then highlight the relationship of the Kuramoto model with neuronal systems at different spatial scales and review the collective behaviors of such systems. One of the main restrictions of Kuramoto's seminal formulation from a neurobiological perspective is its lack of an explicit spatial embedding. In the subsequent section we hence consider two important modifications that incorporate the spatial aspects of neuronal connectivity and the axonal delays that accompany these. Thereafter, we consider less restrictive (second-order) forms of the so-called phase response curves that incorporate the effective coupling between subsystems and consider their candidate physiological counterparts. These lead to the notions of dynamical instabilities and spatial frustrations that arise from the interplay between order and disorder (measured by entropy) in these systems. We illustrate this numerically in two-dimensional corticallike sheets. In the final section, we recast the Kuramoto model at the population level as a particular kind of diffusion process described by the nonlinear Fokker-Planckequation and sketch the insights gained by this formulation. In particular, we review the solutions afforded by this model to recent observations of bistability of the human alpha rhythm and non-Gaussian fluctuations of the beta rhythm in recordings of spontaneous, large scale neocortical activity (Freyer et al., 2009).

\section{SYNCHRONIZATION IN THE KURAMOTO MODEL 2.1 INTRODUCTION TO THE KURAMOTO MODEL}

Like Winfree (1967) before him, Kuramoto sought to understand the collective behavior of a large number of oscillating subsystems, whose states could each be captured by a single scalar phase $\theta$. Such a system can, in general, be represented by the set of $N$ coupled differential equations,

$\dot{\theta}_{n}=\omega_{n}+\sum_{m=1}^{N} \Gamma_{m n}\left(\theta_{m}-\theta_{n}\right), \quad n=1, \ldots, N$,

where the $n$th oscillator, with natural frequency $\omega_{n}$, adjusts its phase velocity according to input from other oscillators through the pairwise phase interaction functions $\Gamma_{m n}$. The natural frequencies $\omega_{n}$ are distributed according to a specified probability density $g(\omega)$ usually taken to be a symmetric, unimodal distribution such as a Lorentzian or a Gaussian with mean $\omega_{0}$. Without loss of generality, the system can be transformed to a rotating frame by subtracting the mean frequency $\omega_{0}$, a helpful convention, which we adopt in the illustrations below.

${ }^{1}$ The Matlab source code for our numerical simulations is available from the authors on request.
The interaction functions $\Gamma_{m n}$ can also be thought of as the phase response of oscillator $n$ to input from $m$. In this formulation, neither the connection topology (e.g., random, lattice, 2D sheet), nor the form of the phase response curve are specified prohibiting specific insights to be obtained. The classic Kuramoto model specifies global (all-to-all) coupling mediated by a sinusoidal interaction function,

$\dot{\theta}_{n}=\omega_{n}+\frac{1}{N} \sum_{m=1}^{N} K_{m n} \sin \left(\theta_{m}-\theta_{n}\right)$

where $K_{m n}$ is a coupling constant. In the homogenous (isotropic) case when the coupling is equal between all pairs of oscillators, i.e., for $K_{n m}=K$ the Kuramoto model reads

$\dot{\theta}_{n}=\omega_{n}+\frac{K}{N} \sum_{m=1}^{N} \sin \left(\theta_{m}-\theta_{n}\right)$

The sinusoidal interaction function is a first-order approximation to the more general form (1) but still permits a variety of highly non-trivial solutions ${ }^{2}$. A notable feature of this choice is that the interaction function vanishes when the phases are identical or differ by $\pi$. In the neighborhood of phase identity the interaction function has the opposite sign of the phase difference between oscillator pairs and hence functions to pull the phases of individual oscillators together. In the case of near-antiphase, the phases are pushed apart, meaning that there exists a single attracting synchronous and a single unstable antiphase constellation for pairs of oscillators. This model is the canonical form for synchronization in extended, oscillatory media.

\subsection{SYNCHRONIZATION AND ORDER PARAMETER FOR THE KURAMOTO MODEL}

Intuitively, the impact of increasing $K$ in the isotropic case should be to increase the phase synchrony amongst the oscillators. This is shown in the first two rows of Figure 1 where we illustrate dynamics for weak, intermediate and strong $K$. In the top row (Figures 1A-C) the phases are visualized on the unit circle in the complex plane whereas the next row (Figures 1D-F) shows brief time series. In the weak case, the oscillators disperse whereas, for strong $K$ they remain relatively synchronous. In the intermediate case, we see that a large cluster of synchronous oscillators are apparent. However, many other oscillators, whose natural frequencies are at the tails of the distribution, are not locked to this cluster. In other words, as $K$ increases, the interaction functions overcome the dispersion of natural frequencies $\omega_{n}$ resulting in a transition from incoherence, to partial and then full synchronization. The phase offset of the fully synchronized solution (approximately $135^{\circ}$ in Figure 1) is determined by the initial phases of the oscillators.

To quantify the degree of synchrony, it is customary to calculate the centroid vector of this phase distribution,

$r e^{i \psi}=\frac{1}{N} \sum_{m=1}^{N} e^{i \theta_{m}}$

${ }^{2}$ The sinusoidal form is "first-order" because it stems from a pair-wise linear coupling between the underlying (self-sustaining) oscillators when approximated by (almost) harmonic balance; see also section 2.3. 
A

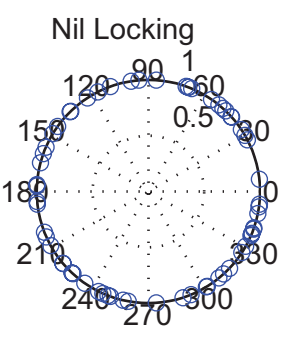

$\mathrm{K} / \mathrm{N}=1$
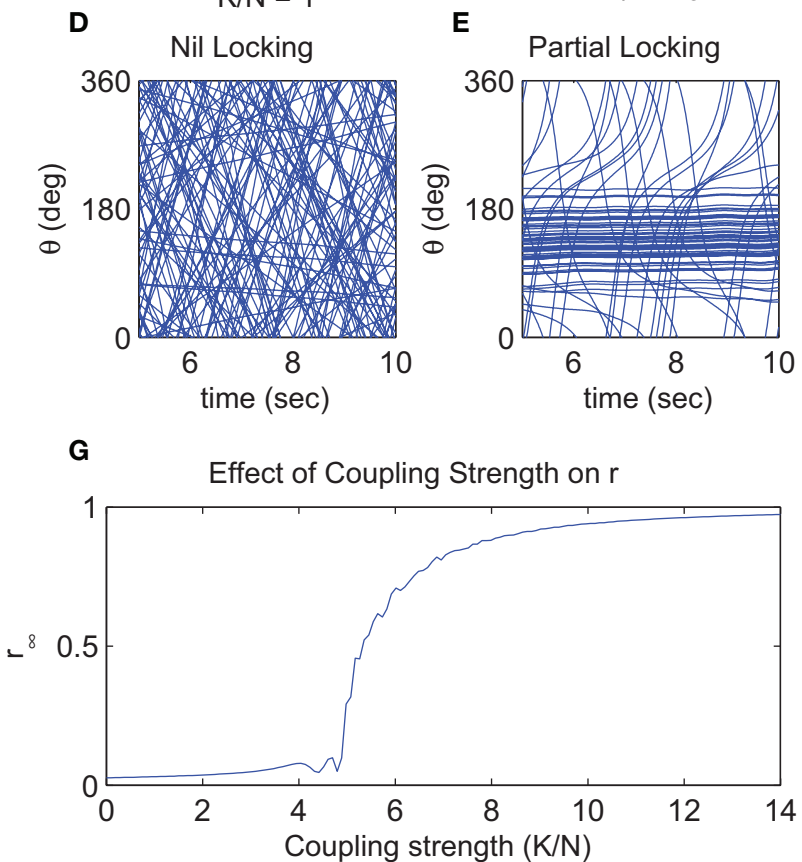

B

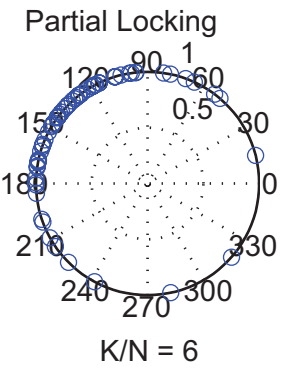

E
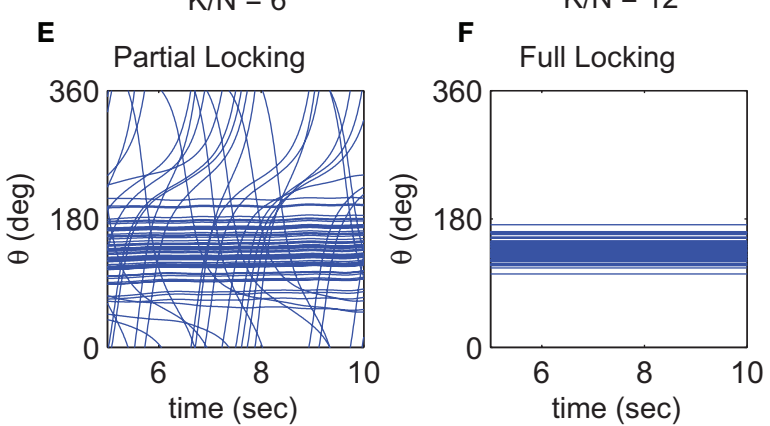

H

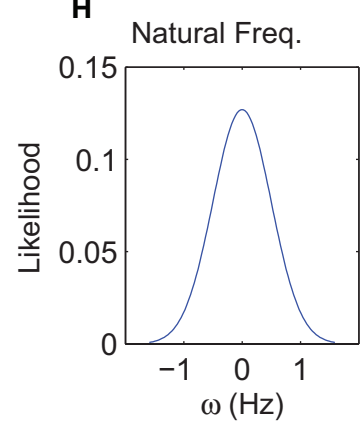

FIGURE 1 | Simulation results for the conventional globally-coupled Kuramoto model $(N=1024)$ under conditions of weak $(K / N=1)$, moderate $(K / N=6)$, and strong $(K / N=12)$ coupling. Top row $(\mathrm{A}-\mathrm{C})$ shows the final phases (in polar form on a unit circle) of the individual oscillators for each condition at $t=10 \mathrm{~s}$. Middle row (D-F) shows the evolution of the oscillator phases during the final $5 \mathrm{~s}$ of each corresponding simulation. For clarity, only the first 64 of the 1024 oscillators are shown. (G) Shows the effect of coupling strength $(K / N=0-14)$ on the phase coherence $\left(r_{\infty}\right)$ of 1024 oscillators at $t=10 \mathrm{~s}$. (H) Shows the Gaussian distribution of natural oscillator frequencies used in these simulations. where $\psi$ is the mean phase of the set of $\theta_{m}$ and the scalar $r$ represents the phase divergence or uniformity (Mardia, 1972). Importantly, $r$ captures the degree of phase coherence in the system as it vanishes when the phases are uniformly distributed (have large circular variance) and approaches one when the phases of all oscillators become aligned. That is, phase coherence $r$ covers the overall structure and is thus identified as the order parameter of the system ${ }^{3}$. Figure $1 \mathrm{G}$ shows the steady-state value of robtained in numerical simulations when the global coupling strength $K$ is manipulated. It can be seen that $r$ remains close to 0 until $K$ reaches a critical value $K_{c}$ (in the figure $K_{c} \approx 5$ ), above which rrapidly increases towards its asymptotic value of 1 . The non-zero values below $K$ merely reflect fluctuations in the simulation due to finite $N$.

${ }^{3}$ The term "order parameter" stems from statistical physics where it is used to quantify different state (or phases) often in terms of thermodynamical potentials like the free energy. In complex dynamical systems, identifying an order parameter relies on a clear-cut separation of time scales: here, $r$ evolves significantly slower than the individual oscillators $\theta_{\text {. }}$. Due to this difference in time scales, all $\theta_{i}$ can quickly adapt to changes of $r$, which thus prescribes the dynamics (or order), it "enslaves" the individual parts of the system (see, e.g., Haken, 1983; Tass, 1999, for more details).
Multiplying both sides of (4) by $e^{-i \theta_{n}}$ and substituting the imaginary parts into (3) recasts the model in terms of the mean field $(\psi, r)$, namely

$\dot{\theta}_{n}=\omega_{n}+K r \sin \left(\psi-\theta_{n}\right)$

This formulation reveals the individual oscillators to be independently enslaved to the mean field alone. Here, circular causality becomes apparent whereby greater phase coherence (larger $r$ ) increases the effective adjustment of each oscillator's phase toward the mean field which thus leads to further increases in phase coherence. Kuramoto exploited this representation to derive an analytic value for $K_{c}$. For instance, if the oscillators' natural frequencies $\omega_{n}$ are distributed around a central frequency $\omega_{0}$ spread by some value $\gamma$ according to a Lorentzian density $g(\omega)=\pi^{-1} \gamma /\left(\gamma^{2}+\left(\omega-\omega_{0}\right)^{2}\right)$, then the critical value reads

$K_{c}=\frac{2}{\pi g\left(\omega_{0}\right)}=2 \gamma$ 
and the dependence of the phase synchrony $r$ on the coupling strength $K$ near the onset of synchrony follows,

$r \approx \sqrt{\frac{16}{\pi K_{c}^{3}}} \sqrt{\frac{K-K_{c}}{K_{c}}\left(\frac{1}{-g^{\prime \prime}(0)}\right)}=\sqrt{\frac{K}{2 \gamma}-1}$.

Equation (6) implies that for a narrow distribution of frequencies around $\omega_{0}$, - i.e., a small $\gamma$ (and hence a high peak $\left.g\left(\omega_{0}\right)\right)-$ synchrony can be achieved for small $K_{c}$. Likewise, $g^{\prime \prime}\left(\omega_{0}\right)$ will be strongly negative and, according to equation (7), $r$ will increase rapidly for $K>K_{c}$. The converse of both large $K_{c}$ and a gradual subsequent increase in $r$ will be true for broad distributions.

Above the critical coupling strength, the completely incoherent state is still a permissible solution to equation (3), but it is unstable (Strogatz and Mirollo, 1991). That is, any perturbation will cause some degree of coherence as reflected in Figure 1G. The incoherent stateloses asymptotic stability, whereas the coherent state becomes attracting. Remarkably, despite the apparently simple form of this system, several crucial properties, such as the stability of the coherent state and the possible existence of other dynamic states, have resisted rigorous proof (see Strogatz, 2000, for a discussion). For example, it was previously known that although the order parameter $r$ decays for $K$ below the critical value $K_{c}$, the incoherent state is only marginally stable in the limit of infinite $N$. Convergence to this state is hence very slow (Strogatz et al., 1992). It was only recently shown that finite size effects introduce strong stability and hence rapid convergence for the incoherent state (Buice and Chow, 2007) although correlations and fluctuations away from this state do persist (Hildebrand et al., 2007). Likewise it has also been recently shown that the partially coherent state is only weakly stable for $K$ above the critical value $K$ and hence also slow to converge (Mirollo and Strogatz, 2007). As the study of synchronous oscillations in neural systems attracts ever more interest, we believe that familiarity between the simplicity of the Kuramoto model and the challenging complexity of its dynamics is an important feature that should warn against simplistic interpretations of experimental signals.

\subsection{RELATIONSHIP TO NEUROBIOLOGICAL SYSTEMS AND INHERENT LIMITATIONS}

Although dynamics restricted to a scalar phase measure for each subsystem may seem highly restrictive, Kuramoto (1984) showed that an ensemble of phase oscillators interacting through an appropriate functional form approximates the long-term behavior of any ensemble of interacting oscillatory systems as long as the coupling is weak and the subsystems nearly identical. This phase reduction approach has now become a standard technique in computational neurosciences (see, e.g., Ermentrout and Kopell, 1986, 1990; Guckenheimer and Holmes, 1990; Tass, 1999; Brown et al., 2004). The phase interaction function (PIF, $\Gamma_{n m}$ ) is itself the convolution of two separate functions, the phase response curve $\left(p_{n m}\right)$ and the perturbation function $\left(z_{n}\right)$ around a full cycle, namely,

$\Gamma_{m n}(\varphi)=\frac{1}{2 \pi} \int_{0}^{2 \pi} z_{n}(\psi) p_{m n}(\psi, \psi+\varphi) d \varphi$.

This formulation is crucially dependent on the oscillators being only weakly coupled, i.e., the mutual perturbations engendered through their interactions are small in comparison to their intrinsic natural frequencies. This permits the interactions to be averaged over a full phase cycle ${ }^{4}$. The functions $z$ and $p$ embody the perturbation of an oscillator away from its intrinsic state due to an input from another (such as via a post-synaptic potential), and the further adjustment in phase as the system returns back to its limit cycle attractor.

The phase reduction approach has afforded a direct link between computational models of neurons and models of weakly coupled phase oscillators, permitting a variety of insights into the relationship between the phase response curve and the nature of synchronous activity at the neuronal level (e.g., Ermentrout, 1986; Ermentrout and Kopell, 1990, 1991; Vreeswijk et al., 1994; Hoppensteadt and Izhikevich, 1997; Kuramoto, 1997). Perhaps the best known example is the elegant reformulation of a simple (class I) spiking neuron as a one dimensional phase oscillator using insights from bifurcation theory (Ermentrout and Kopell, 1986) and the derivation of an appropriate phase response curve (Ermentrout, 1996). Hansel and colleagues (Hansel and Mato, 1993; Hansel et al., 1993a,b) showed that phase-reduced models of weakly excitatory Hodgkin-Huxley neurons elicited comparable phase-locking behaviors as long as the PIF retained at least the first two Fourier components of the original neural interaction; we will return to this in Section 4. They also noticed that the shape of the PIF dictated the overall synchronization properties of the network (Hansel et al., 1995). Neurons with non-negative PIFs failed to synchronize, a property that was later proved true for all class I membrane models (Ermentrout, 1996), whereas those with mixed negative and positive PIFs like Kuramoto's original sine wave formulation, could synchronize. In other words, synchrony is crucially dependent upon oscillators having their phase rotation either advanced or retarded, according to whether it lags or leads the mean field phase, respectively.

Experimental neuroscience, particularly the study of rhythmic behavior in cortical and hippocampal circuits, is increasingly concerned with the activity in large populations of neurons. Phasebased measures of synchrony have become used frequently in the characterization of large-scale experimental neuroscience signals (e.g., Tass et al., 1998; Varela et al., 2001; Breakspear, 2002; Stam et al., 2007; Penny et al., 2009). Computational research into the "mass action" of thousands of neurons has advanced the field of neural mass models (Freeman, 1975). For example, the Wilson-Cowan model describes interacting populations of excitatory and inhibitory neurons and has been widely used in modeling neuronal populations (Wilson and Cowan, 1973). Hoppensteadt and Izhikevich (1997) showed that weakly-coupled Kuramoto oscillators and weakly-coupled Wilson-Cowan oscillators have similar interaction dynamics. They proposed that cortical columns interact through phase modulations - namely that information is carried through periodic modulations of interspike intervals (Hoppensteadt and Izhikevich, 1998). Schuster and Wagner (1990) formally applied the phase-reduction approach to the Wilson-Cowan model and reproduced observations of feature-dependent synchronization between cortical columns in the visual system.

${ }^{4}$ In the study of nonlinear oscillators this averaging is also referred to as "harmonic balance" or as a combination of "rotating wave" and "slowly varying amplitude approximation." 
By specifying the system to be globally connected via purely sinusoidal interaction functions, Kuramoto was able to achieve some crucial analytic insights into oscillatory synchronization, a feat that has been subsequently extended to other important results (e.g., Crawford, 1994). However, to make the system more neurobiologically plausible, some less restrictive assumptions are required with regards to the connection topology and interaction functions. These are covered in the next two sections.

\section{SPATIAL EMBEDDING OF THE KURAMOTO MODEL}

The Kuramoto model specifies global (all-to-all) coupling amongst system oscillators. Whilst this may be a reasonable approximation in a small network of densely connected neurons, it is certainly not true for large populations of neurons distributed across the cortical sheet. In this case, the coupling amongst the oscillators should be spatially embedded. Put differently, it should allow for the presence of time delays between distant subsystems and accommodate reduced coupling strength with distance. We consider each of these in turn.

\subsection{TIME DELAYS, TRAVELING WAVES, AND PHASE FRUSTRATION}

Time delays in neuronal systems arise principally from finite axonal transmission, which is dependent on inter-areal distance and on the presence or absence of myelination as well as on synaptic and dendritic processes. A crucial step toward neurobiological plausibility of coupled oscillators is the incorporation of time delay effects into the PIFs. For a fully-connected Kuramoto model with time delays the dynamics are given by

$$
\dot{\theta}_{n}=\omega_{n}+\frac{K}{N} \sum_{m=1}^{N} \sin \left(\theta_{m}-\theta_{n}-\alpha_{m n}\right)
$$

where $\alpha_{m n}$ translates the time delay $\tau_{m n}$ into a corresponding phase offset. Prior studies that incorporate transmission delays into such networks (Yeung and Strogatz, 1999; Zanette, 2000; Jeong et al., 2002) have revealed elaborate synchronization behaviors. For example, Yeung and Strogatz (1999) incorporated a fixed time delay $\alpha_{m n}=\alpha$ into a fully connected network of Kuramoto oscillators with identical driving frequencies and observed multistable synchrony states as well as a co-existing stable incoherent state. The more complex dynamics due to $\alpha$ suggests the notion of frustration, whereby the interaction functions require some phase offset $\theta_{m}-\theta_{n} \neq 0$ in order to vanish (Acebrón et al., 2005). Put differently, the presence of $\alpha$ causes the interaction functions to pull the phases away from absolute synchrony, even when the natural frequencies are identical. This becomes crucial to the complex dynamics to be explored below. For neurobiological plausibility, it is crucial to order the time delays according to a spatial metric $\alpha_{m n} \propto x_{m}-x_{n} \mid$ either in one dimension or over a two dimensional sheet. Zanette (2000) incorporated distance-dependent transmission delays in a $1 \mathrm{D}$ ring of oscillators and observed a phase transition from global synchrony to propagating spatial wave patterns as the time delay was increased. Similarly, Jeong et al. (2002) observed patterns of global synchrony, traveling rolls, concentric rings, and other spatiotemporal structures in a 2D array of oscillators coupled with distance-dependent delays. Comparable spatiotemporal patterns of firing have been observed in vivo in rabbit (Freeman, 1975), turtle (Prechtl et al., 1997; Lam et al., 2000), cat (Du et al., 2005), and monkey (Grinvald et al., 1994; Arieli et al., 1995, 1996; Rubino et al., 2006) as well as a number of cortical slice preparations observed with rapid-acting voltage-sensitive optical dyes (Roland et al., 2006; Wu et al., 2008). Although the functional importance and role of spatial patterns of oscillatory brain activity have yet to be fully elucidated, spatial patterns of oscillatory activity do have the potential to encode information in their relative spike timing (phase-coding) and hence are worthy of investigation.

The dynamics of globally coupled Kuramoto networks with distance-dependent transmission delays (9) can be approximated by those of zero-delay networks with connection strengths that vary periodically with distance,

$\dot{\theta}_{n}=\omega_{n}+\frac{K}{N} \sum_{m=1}^{N} \cos \left(\alpha_{m n}\right) \sin \left(\theta_{m}-\theta_{n}\right)$,

as described in 7. An example traveling wave solution for this equation is illustrated in Figure 2 (top row). For strong coupling, and uniform natural frequencies, such solutions arise naturally and converge very quickly. Their dominant spatial frequencies coincide with the spatial frequency of $\cos \left(\alpha_{n m}\right)$. The approximation of (9) by (10) only holds exactly when the dynamics are symmetric, as in this case. Traveling wave solutions of (10) are hence stable, attracting solutions of (9), although the transient dynamics towards this global solution will differ. This occurs because the term expressing the difference between these two equations contracts toward zero under the action of sufficiently strong oscillator coupling. The emergence of traveling waves in (10), however, permits a relatively straightforward heuristic. For $K>K_{c}$ this system resembles the original Kuramoto system with the exception that the coupling strength is modulated in magnitude between 0 and 1 , and reversed in sign on intervals of length $\pi$. The coupling strength is maximum in amplitude at $\cos \left(\alpha_{m n}\right)= \pm 1$, corresponding precisely with the wavelength of the traveling waves, and, thus, instances of full pair-wise (anti-) synchrony for all possible oscillator pairs. Between these extremes, there is a phase offset between oscillators that varies directly with the modulation of $K$. That is, the pairs of oscillators relax apart in proportion to the reduction in the effective coupling strength. Hence, in contrast to full synchrony in the original Kuramoto model, the interaction functions do not vanish pair-wise everywhere, but rather their relative contribution to the phase velocity is uniform across the system and globally minimized. Heuristically, the spatial ordering permits a traveling wave dynamic solution that minimizes the frustration, both locally and globally, introduced through the phase offset term $\alpha_{n m}$.

\subsection{FINITE SUPPORT WAVELET-LIKE SPATIAL KERNELS}

Addressing the other neurobiological implausibility of the Kuramoto model, global connectivity can be achieved by combining time delay effects with a finite width spatial kernel: a function that is maximum centrally and reduces gracefully toward zero at some finite width. The convolution of a periodic function with such a kernel yields a wavelet-like modulation of the interaction functions,

$\dot{\theta}_{n}=\omega_{n}+\frac{K}{N} \sum_{m=1}^{N} W(m, n) \sin \left(\theta_{m}-\theta_{n}\right)$, 
where $W(m, n)$ is such a function. The lower row of Figure 2 illustrates an example employing the fourth derivative of a Gaussian function as an example kernel. As with (10), traveling wave-like structures emerge in this system. More specifically, traveling solutions emerge locally with spatial frequencies that conform to those of the spatial kernel. However, because the periodically modulated phase interactions are only imposed locally, not globally, the system converges only slowly toward these solutions and fluctuates strongly en route. Moreover, even if the natural frequencies of the oscillators are uniform, regions of sudden phase stress appear at sporadic spatial locations. Examples, such as near oscillator \#60, can be seen in Figure 2. In two dimensions, as shown in Figure 3, such points appear near complex intersections between coherent fronts of traveling waves. Their locations change but the existence of these points persists, corresponding to collisions between the local phase-coherent structures. The presence of local traveling structures at most locations reduces the expression of the PIFs across these domains. In keeping with our heuristic above, the sporadically occurring strong phase reversals reflects small regions where they are expressed strongly because of the influence of phase incongruent waves on either side of these points. This leads to isolated phase rotations that diverge strongly from the local natural frequency.


FIGURE 2 | Spatial patterns of phase locking in the 1D Kuramoto model $(n=128)$ at convergence $(t=10 \mathrm{~s})$ under conditions of global versus local synaptic kernels. Top row shows the spatial pattern of phase locking adopted by the oscillators (left panel) when coupled using a cosine-with-distance kernel which extends to infinity las shown in the top-right panel). The bottom-left panel shows the spatial pattern of phase locking evoked by a local kernel corresponding to the fourth derivative of a Gaussian (as shown in the bottom-right panel). Initial conditions were identical in both cases and natural frequencies were normally distributed as per Figure 1.
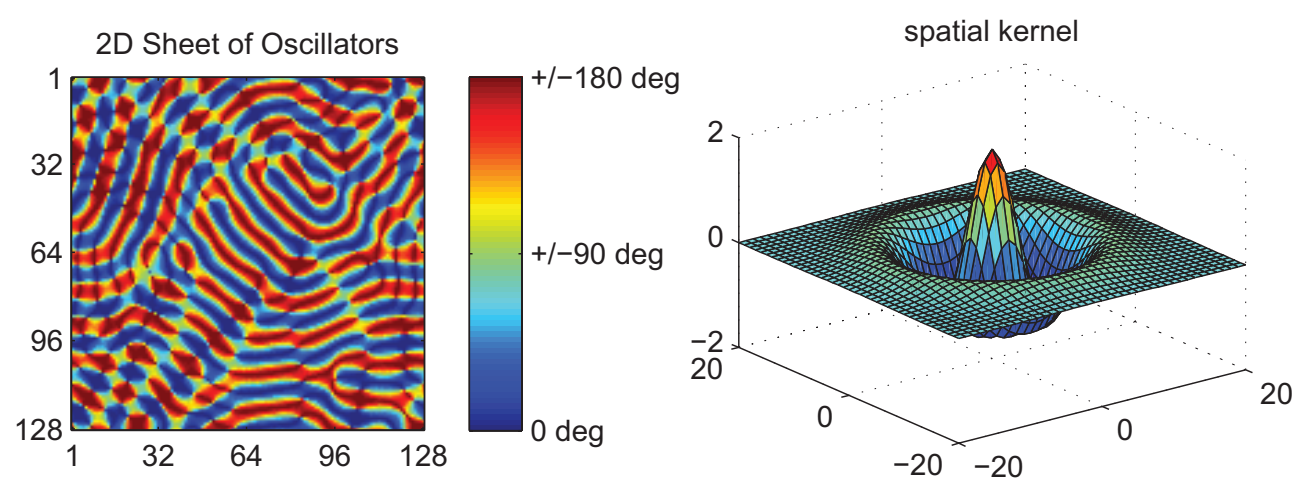

FIGURE 3 | Spatial patterns of phase-locking in the 2D Kuramoto model $(128 \times 128)$ using a local kernel corresponding to the fourth derivative of the Laplacian of the Gaussian. As before, the natural oscillator frequencies were normally distributed $(M=0 \mathrm{~Hz}, \mathrm{SD}=0.5 \mathrm{~Hz})$. 
Put differently, confining the periodic modulation of the PIFs to local domains, means that the frustration introduced through the phase offset term is reduced by traveling wave structures globally, but not locally everywhere.

It is straightforward to calculate the divergence between the natural frequencies and the oscillator frequencies,

$F_{n}=\omega_{n}-\frac{K}{N} \sum_{m=1}^{N} W(m, n) \sin \left(\theta_{m}-\theta_{n}\right)$,

either locally, or integrated across the entire domain $n=1, \ldots, N$. Numerical simulations show that this global quantity invariably decreases strongly as the system traverses from random initial conditions toward such solutions although it increases markedly at the points of spatial incoherence. We believe that local traveling wave structures, which confine the expression of phase frustration to small, isolated locations, represent a globally optimal minimum to this function although we do not provide a proof for this assertion. Were this to be the case, the dynamics (12) could be recast as a gradient descent on the free energy of the system, namely the divergence between the expected pair-wise phase alignment expressed a priori by the coupling function on the r.h.s. of (11) and the dynamical solutions observed a posteriori.

We explored a wide range of spatial kernels that combined a finite effective spatial support with an oscillatory component. Traveling structures emerge on a wide variety of these. Domains of well formed waves typically appear when the outermost extent of these kernels is negative (phase retarding) whereas large coherent slow moving fronts, often organized in spiral formations, arise when the outermost front is positive.

\section{PHASE RESPONSE CURVES, COMPLEX DYNAMICS, AND ENTROPY}

Kuramoto's original formulation of the interaction function (8) as a single sinusoidal function with zero phase offset results from a truncation of a Fourier expansion of this $2 \pi$-periodic function to the first mode. As we noted, the presence of a phase advancing and phase retarding region around the zero crossing is crucial to synchronization. However, because this function is itself the con- volution of two distinct physiological processes, a more complex form would arguably provide greater capacity for it to represent these underlying processes and their modulation.

\subsection{DYNAMICAL INSTABILITIES DUE TO SECOND ORDER PHASE INTERACTION CURVES}

Hansel and colleagues (Hansel and Mato, 1993; Hansel et al., 1993a,b) showed that phase-reduced models of weakly excitatory, synaptically coupled Hodgkin-Huxley neurons elicited comparable phase-locking behaviors as long as the PIF retained at least the first two Fourier components of the original neural interaction. They employed the form,

$\Gamma(x)=-\sin (x+\beta)+R \sin (2 x)$,

where $R$ is a scalar that modifies the contribution of the second order term and $\beta$ de-phases the relative position of the two modes. This adjustment allows the PIF to incorporate extra biophysical detail such as specific forms of post-synaptic currents (Hansel et al., 1995). The first thing to note is that the two modes have opposite sign: whereas the first term, as in the Kuramoto model, pulls the phases toward $\beta$, the second term tends to destabilize this phase configuration. When $\beta>0$ there exists $R>R_{c}(\alpha)$ for which these two modes intersect twice and therefore the PIF vanishes at four points along a full cycle: two stable (attracting) nodes separate two unstable saddles. These extra fixed points therefore facilitate the existence of distinct clusters of phase locked oscillators even if the coupling is otherwise global, a phenomenon that is not possible with the first Fourier mode alone. As illustrated in Figure 4, if $R$ decreases the two extra fixed points of the PIF approach each other and then annihilate in a saddle-node bifurcation as $R$ crosses below $R_{i}$.

This dynamic instability in the PIF enables a rich variety of more complex dynamics, most notably the emergence of heteroclinic cycles (Hansel et al., 1995). In essence, the presence of a saddle point between the cluster states engendered by the second order PIF allows for spontaneous cycling between different phase synchronous configurations. That is, in a network of $N$ oscillators there may exist $k$ distinct clusters each of size
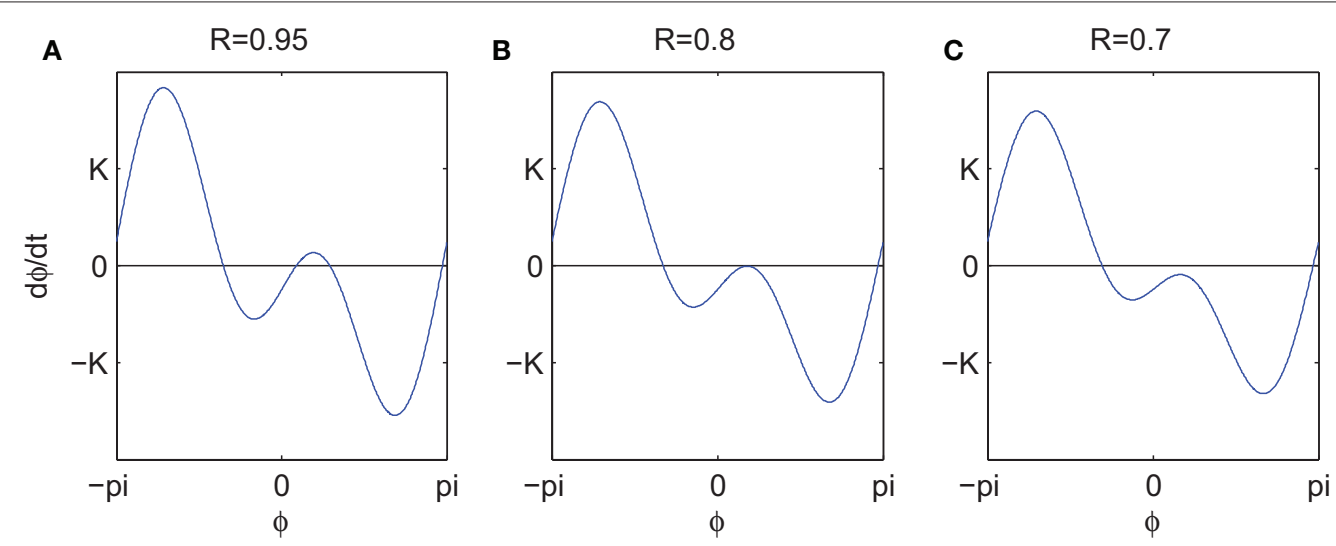

FIGURE 4 | (A-C) Show the second order phase interaction employed by Hansel and colleagues with $\boldsymbol{\beta}=\mathbf{0 . 2 5}$ and three values of $\boldsymbol{R}$. A saddle-node bifurcation in the fixed points associated with this function occurs as the second peak in this curve crosses 0 (left to right). 
$m_{1}, m_{2}, \ldots, m_{k}$ (e.g., Tass, 1999). Through the presence of the saddle point, however, one or more of these cluster states is only marginally stable meaning that their oscillators spontaneously de-phase. These free oscillators then synchronize with other clusters, destabilizing at least one of these so that this winnerless competition continues ad infinitum. Whilst this occurs in the absence of noise, the injection of a stochastic influence into the states stabilizes the frequency of the slow heteroclinic cycling which then scales with log of the variance noise (Hansel et al., 1993a).

We note that in these systems, two distinct time scales arise naturally: the fast dynamics of the oscillators and the relatively slow rotation through the heteroclinic cycle. Systems with distinct time scales have been well studied as they often arise through mere construction, e.g., multiplication of one or more dynamical variables by an explicit time scale factor which functions to slow down the dynamics in the associated subspace (e.g., Fujimoto and Kaneko, 2003; Breakspear and Stam, 2005; Kiebel et al., 2008). By contrast, a heteroclinic cycle does not require a separation of time scales to be defined in functional form because they are an emergent property of the dynamics. The intricate sequence of cycle states and the controlled expression of instability allows a variety of putative computational functions to be enacted by such networks, even with relatively small number of oscillators. For example, Ashwin et al. (2007) showed that procession through a precisely defined cycle could function to encode a complex, sensory input as a spatiotemporal sequence in a manner that was robust to strong noise (Wordsworth and Ashwin, 2008) and could be learnt by other systems of coupled oscillators (Orosz et al., 2009).

\subsection{DYNAMICAL INSTABILITIES AND SPATIAL FRUSTRATION ON CORTICAL-LIKE SHEETS}

By combining the spatial coupling discussed in Section 3 with the second-order phase interaction function, it is possible to employ the framework of weakly coupled oscillators to understand spontaneous dynamics on cortical-like sheets, an area of strong current interest (Fox and Raichle, 2007; Honey et al., 2007; Deco et al., 2009). In particular, it is possible to explore the relationship between the dynamic instability engendered by the interaction of the two modes of the PIF and the spatial expression of frustration introduced by the phase offset. In Figure 5, we present three simulations employing the PIF defined by equation (13) and increasing the de-phasing of the PIF modes by increasing $\beta$. Following Hansel and Mato (1993), we fix the relative modulation of the PIF by the second mode to $R=0.25$. Phenomena such as clustering and cycling are robust to changes in this value. In the top row (Figures $5 \mathrm{~A}-\mathrm{C}$ ), we plot a snapshot of the relative phases and in the bottom row (Figures 5D-F), the local expression of the coupling influence given by equation (12). In the absence of significant de-phasing $(\beta \approx 0)$ the system evolves rapidly toward spatiotemporal patterns dominated by coherent fronts of traveling waves with small pinwheellike patterns where these intersect (Figure 5A). Local expression of high phase frustration is apparent at these points (Figure 5D).

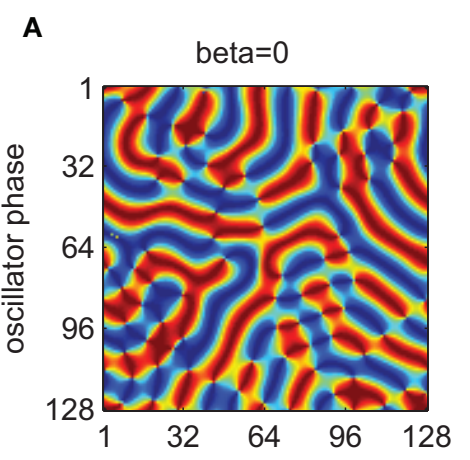

B
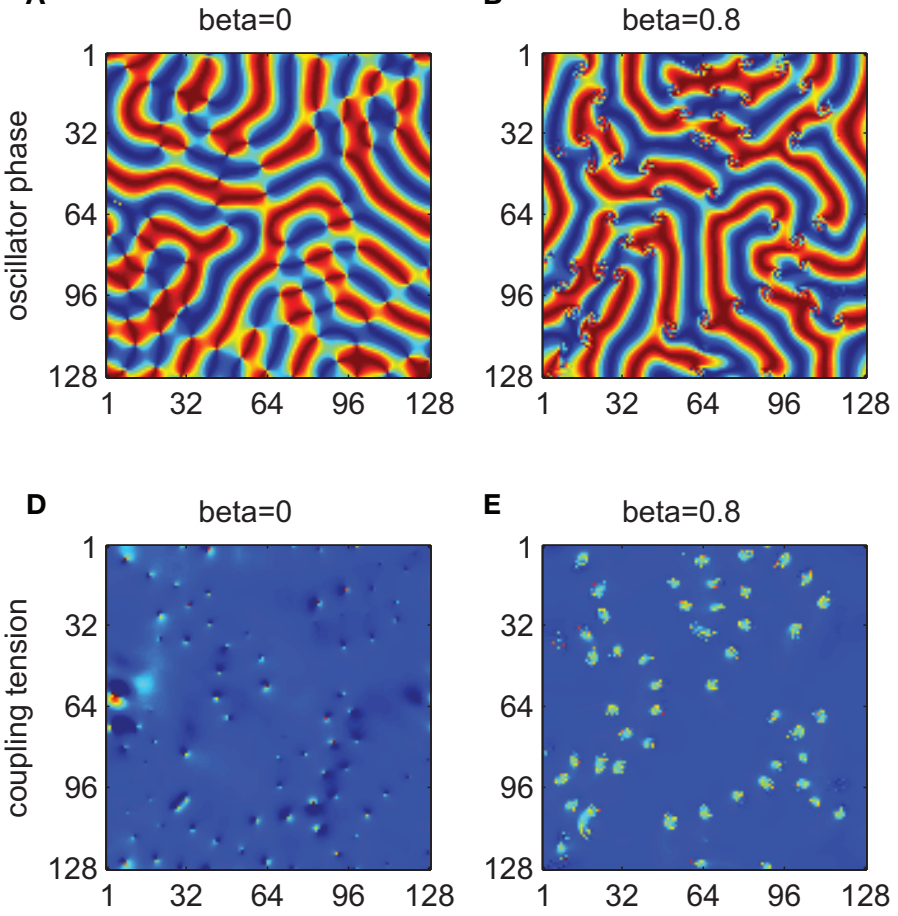

E



C

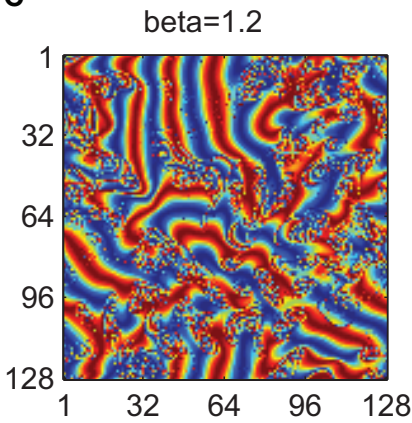

$\mathbf{F}$

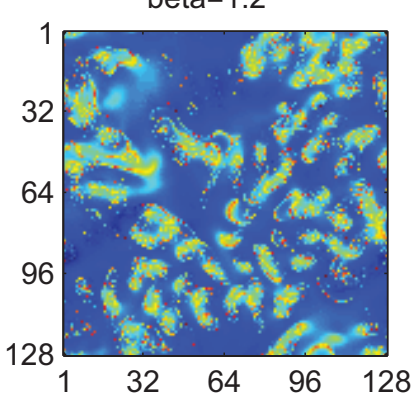

FIGURE 5 | Spatiotemporal dynamics in systems of oscillators coupled through a local spatial kernel and a second order PIF, equation (13). Columns left to right depict results for increasing phase offset $\beta$ between the two modes. Top row $(\mathbf{A}-\mathbf{C})$ shows representative oscillator states using the same color scheme as Figure 3. Bottom row (D-F) shows the coupling tension $F$ as defined by equation (12) where blue denotes $F=0$. 
Hence, without de-phasing between the first and second modes, the scenario is almost identical to that encountered with the spatial kernel and simple sinusoidal PIF (Figure 4). However, as $\beta$ slowly increases, an instability appears at these points and grows to encompass a small patch of oscillators (Figures 5B,E). With a further increase of $\beta$ these instabilities grow in spatial extent and, whilst not evident in a snapshot, begin to invade the surrounding patches of coherent wave fronts (Figures 5C,F). In consequence, the instability in the PIF, introduced by a de-phasing of the first and second Fourier modes, is inconsequential in areas of low spatial frustration. However, at points of spatial incoherence, this dynamic instability leads to a spatial instability expressed as areas of high phase disorder. This effect appears to be invariant to particular choices of the spatial kernel. In Figure 6, we illustrate an example using a spatial kernel whose outer extent is phase advancing, and which is associated with large traveling fronts organized around pinwheels. With a de-phasing of the two modes of the PIF, the centers of the pinwheels are destabilized by the same tension between phase frustration and the dynamic instability within the PIF.

\section{POPULATION-LEVEL DESCRIPTORS OF CORTICAL RHYTHMS}

In Section 2, we introduced the notion of the order parameter $r$ for the Kuramoto model and showed how the governing equation could be rewritten using this quantity. In many instances, however, one is not only interested in the mean field, i.e., the mean phase $\psi$ and its divergence $r$, but also in the nature of the whole probability distribution of states (for review, see Deco et al., 2008), or at the least its first few moments (mean, variance, skewness, kurtosis).
This is particularly true when seeking to establish a link between cortical dynamics and cognitive processes because there is a natural mapping between the moments of neuronal states and components of cognition, including expectation, certainty, and surprise (Friston and Dolan, 2009). The distribution of states is also a crucial notion when the oscillators are influenced by stochastic forces. Finally, the probability distribution is crucial to a proper understanding of data obtained from oscillating neuronal systems because it determines the moment-to-moment statistics of these time series as the underlying system randomly samples its phase space.

In this section, we briefly overview the population formulation of the Kuramoto model, namely the nonlinear Fokker-Planck equation, and contrast it to the linear Fokker-Planck equation that can be derived from populations of spiking neurons. This is a crucial aspect of the Kuramoto model because it nicely recasts the circular causality that is present in the original formulation, whilst also underlining many of the important analytic results discussed in Section 2 (e.g., Strogatz and Mirollo, 1991; Acebrón et al., 2001). By knowing the distribution of states, it is also possible to estimate information-theoretic quantities such as entropy and hence provide a more direct link to notions of free energy described in Sections 3 and 4 .

Before proceeding, it is crucial to underline an important distinction between the probability distribution of the states of the oscillators $p$ on the one hand, and the population density of the whole ensemble $\rho$ on the other. The population density is a quantitative measure of the relative states of all the oscillators and can be estimated in large purely deterministic systems as well as
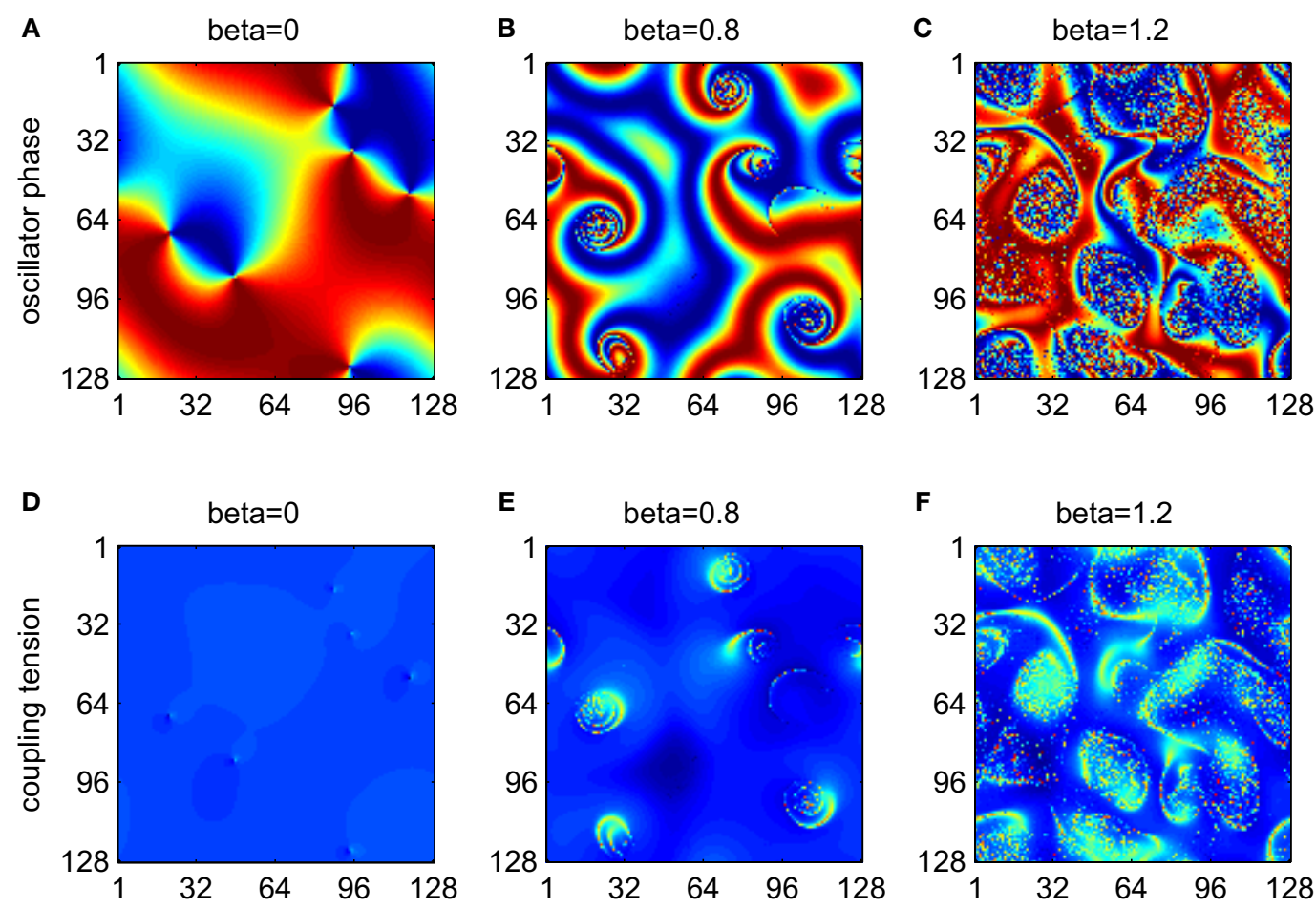

FIGURE 6 |As with Figure 5 with the exception of a larger spatial kernel whose outer extent is upgoing (phase advancing) hence engendering large coherent fronts and spiral waves. 
those that have explicit stochastic forces operating on the oscillators. The probability distribution $p$ is the likelihood function for oscillators and only makes sense when stochastic influences have been explicitly defined. In some, quite general cases, the two are interchangeable. In the following, we first consider the population density of states in a large ensemble of Kuramoto oscillators because it follows naturally from the preceding focus on deterministic dynamics. We then introduce the stochastic Kuramoto model and consider the evolution of the probability distribution that can defined in this setting. Finally, we consider the interchangeability of $p$ and $\rho$ in large stochastic Kuramoto oscillators.

\subsection{THE CONTINUITY EQUATION}

We first consider the population density formulation of the pure Kuramoto model. In the so-called thermodynamic limit of an infinite number of oscillators the mean field centroid vector, equation (4), can be written as an average over the phases and frequencies of the ensemble,

$$
r e^{i \phi}=\int_{-\infty}^{+\infty} e^{i \theta} \rho(\theta, t) d \theta
$$

A continuity equation can be established by noting that any change in the shape of the population density, due to the drift $v_{n}$ of any one or more oscillators, is governed by the Kuramoto model. Averaging over the pre-specified frequency distribution $g(\omega)$ simplifies the Kuramoto model to deviations from the mean frequency $\omega_{0}$. Then the continuity equation reads

$\frac{\partial \rho}{\partial t}+\frac{\partial}{\partial \theta}\left\{\left[\omega_{0}+K r \sin (\psi-\theta)\right] \rho\right\}=0 ;$

for the sake of generality we here use a non-vanishing mean frequency $\omega_{0}$.

This equation simply restates the Kuramoto model at the level of the entire population, and demands that perturbations of this function must obey the underlying deterministic equations whilst also preserving the area under the density curve. In the thermodynamic limit, significant fluctuations around the mean field vanish. However, in the finite size setting, there exists a precise, hierarchical organization of all higher order moments which scale with $1 / N$, inversely with system size (Hildebrand et al., 2007). The nature and role of fluctuations in neuronal oscillations - discussed further below - underlines the importance of a full understanding of these moments.

\subsection{STOCHASTIC FORCES AND THE FOKKER-PLANCK EQUATION}

All the dynamics discussed thus far incorporate stochasticity solely through the randomness of the oscillators' frequencies $\omega_{n}$. Now we include stochastic forces by explicitly introducing white noise into the dynamics (3). In order to do so, we return to the finite $\mathrm{N}$ ensemble of phase oscillators and write the so-called Langevin equation of the stochastic Kuramoto model,

$\dot{\theta}_{n}=\underbrace{\omega_{n}+\frac{K}{N} \sum_{m=1}^{N} \sin \left(\theta_{m}-\theta_{n}\right)}_{v_{n}}+\xi_{n}(t)$. where the $\xi_{n}(t)$ are spatially independent and temporally uncorrelated random fluctuations with vanishing means and variance $\sigma^{2}$. These fluctuations may arise from influences that are external to the system, or they may represent a correction to the incomplete specification of a complex system as a system of weakly coupled oscillators. Naturally, in real systems, modeling such fluctuations is crucial as they inevitably occur even if only because of thermal effects. Indeed, functional forms which introduce and explicitly parameterize stochastic processes in real neuronal systems are arguably of great importance because of the mounting evidence for the functional role of noise in neurophysiological recordings (Faisal et al., 2008), perceptual performance (Moss et al., 2004) and computational models of the brain (Deco et al., 2009).

The dynamics of the joint probability of the distinct oscillators' states, $p\left(\theta_{1}, \theta_{2}, \ldots, \theta_{N}, t\right)$, embody a diffusion process as the stochastic fluctuations $\xi_{n}$ lead to divergence and hence a finite, non-zero variance in the ensemble. The diffusion supplements the deterministic, intrinsic forces caused by the interaction between the oscillators, as discussed above. The resulting mathematical form for the timeevolution of the $p$ is referred to as the Fokker-Planck equation of the system (Stratonovich, 1963; Risken, 1989). In fact, there is a variety of possibilities to derive the Fokker-Planck equation for the stochastic Kuramoto model (e.g., Sakaguchi, 1988; Sakaguchi et al., 1988). Without going into detail, for (16) one finds

$\frac{\partial p}{\partial t}=\sum_{n=1}^{N}\left(-\frac{\partial}{\partial \theta_{n}}\left[v_{n} p\right]+D \frac{\partial^{2} p}{\partial \theta_{n}^{2}}\right)$,

where the scalar $D=1 / 2 \sigma^{2}$ parameterizes the amplitude of the stochastic forces ${ }^{5}$.

As discussed earlier, the Kuramoto model can be recast using its mean field (5). In the presence of stochastic forces, this approximation becomes

$\dot{\theta}_{n}=\omega_{n}+K r \sin \left(\psi-\theta_{n}\right)+\xi_{n}(t)$.

When averaging over the frequency distribution $g(\omega)$ of the natural frequencies, the dynamics reduces to

$\dot{\theta}=\omega_{0}+K r \sin (\psi-\theta)+\xi_{n}(t)$,

which finally yields a simpler form of the Fokker-Planck equation, namely

$\frac{\partial p}{\partial t}=-\frac{\partial}{\partial \theta}\left\{\left[\omega_{0}+K r \sin (\psi-\theta)\right] p\right\}+D \frac{\partial^{2} p}{\partial \theta^{2}}$

where $r$ is given by (14). If the variance of the noise goes to 0 , then the probability density $p$ is replaced by the population density $\rho$ and this second-order partial differential equation reduces to the first-order continuity equation (15).

${ }^{5} \mathrm{~A}$ typical strategy to solve this equation is first to look a the corresponding marginal distributions, e.g., $p\left(\theta_{1}, \ldots, \theta_{j-1}, \theta_{j+1}, \ldots, \theta_{N}, t\right)=\int_{-\pi}^{+\pi} p\left(\theta_{1}, \theta_{2}, \ldots, \theta_{N}, t\right) d \theta_{j}$, by which the $N$-dimensional state space can be iteratively reduced step-wise. Exploiting the equivalence between the different phases, then integrating (17) over all but one state space variables yields (by approximation) the dynamics of $p(\theta, t)$ as $(23)$ below. This procedure is closely related to a mean-field approach (see Sakaguchi, 1988; Sakaguchi et al., 1988, for more details). 


\subsection{THE NONLINEAR FOKKER-PLANCK EQUATION}

The quantity $\theta$ does not only describe all possible states but is also a representative of all individual phases - that is, phases are indistinguishable because the population has been represented as homogeneous. Put alternatively, the temporal average over states converges to the spatial average over the ensemble because the dynamics are mixing (ergodic). When this assumption holds, then we can readily identify the density $\rho(\theta, t)$ of "real" oscillators $\theta_{1}, \theta_{2}, \ldots$, discussed in Section 5.1 , with the probability density $p(\theta, t)$. That is, we use

$r e^{i \psi}=\int_{-\pi}^{+\pi} e^{i \theta} p(\theta, t) d \theta$.

Substituting this into (19) yields

$\dot{\theta}=\omega_{0}+K \int_{-\pi}^{+\pi} \sin (\vartheta-\theta) p(\vartheta, t) d \vartheta+\xi(t)$

here we again averaged over the frequency distribution. Following Frank et al. (2000) we write the Fokker-Planck equation as

$\frac{\partial p}{\partial t}=-\frac{\partial}{\partial \theta}\left\{\left[\omega_{0}+K \int_{-\pi}^{+\pi} \sin (\vartheta-\theta) p(\vartheta, t) d \vartheta\right] p\right\}+D \frac{\partial^{2} p}{\partial \theta^{2}}$.

Whilst this form is indeed similar to (20), by explicitly incorporated the mean field into the dynamics, it is straightforward to see that the Fokker-Planck equation is non-linear in its probability density ${ }^{6}$.

The second term on the r.h.s. of (23) incorporates the tendency of the stochastic influences to scatter the density toward a uniform distribution around the unit circle. If $K$ equals 0 then the first term vanishes and this is all that occurs. The first term embodies the deterministic tendency of the coupling amongst the oscillators to increase the density around the mean of the natural frequencies $\omega_{0}$. The observation that this term is nonlinear in the density of states $p$ is nothing other than a recasting of the circular causality we encountered in Section 2: As the system synchronizes, the density of states contracts, at the same time increasing the effective pull toward the mean. In the presence of stochastic influences, the aggregation of the members of the ensemble toward the minimum of this energy landscape, through the deterministic force is amplified by means of statistical feedback. That is, the more probable a stable state, the less it is affected by noise and, conversely, the less a stable state may be affected by noise, the more probable it is (Frank et al., 2002). This contrasts strongly with traditional accounts of diffusion under a constant force, such as regular diffusion in a harmonic potential, where the force is imposed externally and constant. Even in the presence of strong noise, it means the system can depart from classic exponential statistics, showing a tendency toward power-law scaling in the character of its temporal fluctuations (Sokolov et al., 2002; Zaslavsky, 2002). Its characterization requires novel tools from nonextensive thermodynamics like generalized entropies (Tsallis and Brigatti, 2004; Tsallis, 2006).

${ }^{6}$ This should not be confused with conventional Fokker-Planck equations whose $\mathrm{drift}$ and diffusion coefficients depend non-linearly on the state space. These equations are linear in $p$, whilst (23) is not.

\subsection{CONTRASTING LINEAR AND NON-LINEAR FOKKER-PLANCK EQUATIONS}

It is likewise possible to derive a Fokker-Planck representation of ensembles of spiking neurons (e.g., Deco et al., 2008), although doing so typically requires the diffusion assumption, namely that the currents arising at individual neurons are uncorrelated. This step allows higher order moments and their coupling to be discarded and leads to the drift term of the r.h.s. in (17) being linear in both the states and the density. This contrasts with the derivation of the Fokker-Planck equation for the Kuramoto model for which correlations amongst the inputs are an indispensable property of the model, and for which, through the nonlinearity in the density, the moments of the ensemble are interdependent. The linear Fokker-Planck equation for spiking neurons describes a process that is akin to classic diffusion in the presence of an external force and hence predicts that the distribution of the states should be approximately Normal and the spikes Poisson. The sufficient statistics in this setting include just the mean and the variance. The diffusion assumption is certainly consistent with a powerful body of research that posits a crucial role for these Gaussian statistics in the performance of optimal Bayesian inference through population coding (Ma et al., 2006; for review, see Friston and Dolan, 2009). Several recent papers have reported that the statistics of non-rhythmic activity at high frequencies (above $30 \mathrm{~Hz}$ ) are consistent with a classic Poisson process (Bedard et al., 2006; Miller et al., 2009). Crucially, this corresponds to activity across a broad frequency range of non-rhythmic activity that has a featureless power spectral density.

The nonlinear Fokker-Planck equation allows for a departure from these classic processes. Specifically, through an interaction between the density of the states and the effect of stochastic influences, the nonlinear Fokker-Planck equation allows for a partially synchronized system to exhibit long dwell times near complete synchrony and associated extremal amplitude events in the mean field term, properties that have recently been documented in the beta $(\approx 20 \mathrm{~Hz})$ rhythm of human resting state EEG (Freyer et al., 2009). These non-classic statistics in the amplitude fluctuations supplement prior findings of longtailed distributions in the temporal statistics of low frequency (below $30 \mathrm{~Hz}$ ) rhythmic cortical activity (Linkenkaer-Hansen et al., 2001; Stam and de Bruin, 2004). Activity at lower frequencies showing non-classic statistics is associated with clear peaks in the power spectrum that are hence consistent with an underlying rhythmic process, in contrast to feature-less high frequency activity.

Another interesting departure of the nonlinear FokkerPlanck equation from classic statistics is its ability to support the coexistence of multiple co-occurring attractor states - or multistability (Frank et al., 2000). In the presence of either sufficiently large stochastic influences, or marginal attractor stability, this allows a system of coupled oscillators to erratically and spontaneously switch between different itinerantly expressed solutions. This is also of particular relevance for cortical rhythms, given the recent evidence for such bimodality in the human alpha rhythm (Freyer et al., 2009). In particular, the alpha rhythm appears to spontaneously and erratically 
switch between a low and high amplitude state, consistent with noise-driven switching between co-existing coherent and incoherent phase configurations amongst the underlying oscillators. It also suggests a unifying mechanism for multistability that has been observed in a number of basic cortical functions including human perception (Ditzinger and Haken, 1989), decision making (Deco and Rolls, 2006), and behavior (Schoner and Kelso, 1988).

In summary, whilst rhythmic behavior appears to conform with the anomalous statistics of the nonlinear Fokker-Planck equation, non-rhythmic behavior appears to be consistent with uncorrelated spiking activity that conforms to the diffusion approximation. At this stage, apart from a somewhat unaesthetic partition of cortical activity into distinct correlated rhythms and uncorrelated broad frequency spiking activity, it is difficult to see how this apparent paradox can be reconciled.

\section{CONCLUSION}

The objective of the present manuscript was to provide a neurobiologically minded overview of the essential concepts, dynamics, and analysis of the Kuramoto model, which can be considered a canonical model of synchronous oscillations in complex systems. In the purely deterministic setting, we traced the impact of introducing interaction functions and spatial embeddings that may be more representative of neuronal processes. Even in the absence of random forces, we saw that these introductions engendered a broad repertoire of rich, non-trivial dynamics. Although the framework becomes mathematically more challenging, introducing stochastic influences and studying population-wide responses is still possible.

\section{REFERENCES}

Acebrón, J., Bonilla, L., Pérez Vicente, C., Ritort, F., and Spigler, R. (2005). The Kuramoto model: a simple paradigm for synchronization phenomena. Rev. Mod. Phys. 77, 137-185.

Acebrón, J., Perales, A., and Spigler, R. (2001). Bifurcations and global stability of synchronized stationary states in the Kuramoto model for oscillator populations. Phys. Rev. E64, 16218.

Arieli,A., Shoham, D., Hildesheim, R., and Grinvald, A. (1995). Coherent spatiotemporal patterns of ongoing activity revealed by real-time optical imaging coupled with single-unit recording in the cat visual cortex. J. Neurophysiol. 73, 2072-2093.

Arieli, A., Sterkin, A., Grinvald, A., and Aertsen, A. (1996). Dynamics of ongoing activity: explanation of the large variability in evoked cortical responses. Science 273, 1868-1871.

Ashwin, P., Orosz, G., Wordsworth, J., and Townley, S. (2007). Dynamics on networks of cluster states for globally coupled phase oscillators. SIAM J. Appl. Dyn. Syst. 6, 728-758.

Bedard, C., Kroeger, H., and Destexhe, A. (2006). Does the $1 /$ f frequency scaling of brain signals reflect self-organized critical states? Phys. Rev. Lett. 97, 118102.
Biswal, B., Yetkin, F., Haughton, V., and Hyde, J. (2005). Functional connectivity in the motor cortex of resting human brain using echo-planar MRI. Magn. Reson. Med. 34, 537-541.

Boonstra, T., Daffertshofer, A., Breakspear, M., andBeek,P.(2007).Multivariatetimefrequency analysis of electromagnetic brain activity during bimanual motor learning. Neuroimage 36, 370-377.

Breakspear, M. (2002). Nonlinear phase desynchronization in human electroencephalographic data. Hum. Brain Mapp. 15, 175-198.

Breakspear, M., and Stam, C. (2005). Dynamics of a neural system with a multiscale architecture. Philos. Trans. R. Soc. Lond., B, Biol. Sci. 360, 1051.

Bressler, S., and Freeman, W. (1980). Frequency analysis of olfactory system EEG in cat, rabbit, and rat. Electroencephalogr. Clin. Neurophysiol. 50, 19-24.

Brown, E., Moehlis, J., and Holmes, P. (2004). On the phase reduction and response dynamics of neural oscillator populations. Neural Comput. 16, 673-715.

Buice, M., and Chow, C. (2007). Correlations, fluctuations, and stability of a finite-size network of coupled oscillators. Phys. Rev. E 76, 31118.

When written in this manner, we see the essential component of the Kuramoto model is preserved, namely the tendency of synchronization to become self-fulfilling, buffering the mean field against the internal noise.

We have argued that computational neuroscience can benefit from detailed physiological models at all spatial and temporal scales as well as more abstract approaches that seek deeper unifying mechanisms. This is an approach that has led to many exciting discoveries in the physical sciences that are able to unify apparently diverse phenomena. It is equally true that understanding the role of detailed mechanisms provides deeper insight into their precise mechanistic and functional roles. One challenge facing both these fields is to unify the apparently uncorrelated character of noisy spike trains, with the correlated and non-Gaussian nature of rhythmic dynamics.

We hope that the rich dynamics arising from this simple system inspires computational neuroscientists interested in the fundamental mechanisms of cortical rhythms to further investigate its historical and conceptual foundations.

\section{ACKNOWLEDGMENTS}

We thank Kees Stam, Pete Ashwin, Angela Langdon, and Tjeerd Boonstra for formative discussions on the Kuramoto model and the role of oscillations in cortical systems. Michael Breakspear and Stewart Heitmann acknowledge the support of ARC Thinking Systems support and Brain Sciences UNSW. Michael Breakspear acknowledges the support of BrainNRG. Andreas Daffertshofer thanks the Netherlands Organisation for Scientific Research (NWO) for financial support.

Crawford, J. (1994). Amplitude expansions for instabilities in populations of globally-coupled oscillators. J. Stat. Phys. 74, 1047-1084.

Deco, G., Jirsa, V., McIntosh, A., Sporns, O., and Kötter, R. (2009). Key role of coupling, delay, and noise in resting brain fluctuations. Proc. Natl. Acad. Sci. U.S.A. 106, 10302.

Deco, G., Jirsa, V., Robinson, P., Breakspear, M., and Friston, K. (2008). The dynamic brain: from spiking neurons to neural masses and cortical fields. PLoS Comput. Biol. 4. doi: 10.1371/ journal.pcbi.1000092.

Deco, G., and Rolls, E. (2006). Decisionmaking and Weber's law: a neurophysiological model. Eur. J. Neurosci. 24, 901-916.

Ditzinger, T., and Haken, H. (1989). Oscillations in the perception of ambiguous patterns: a model based on synergetics. Biol. Cybern. 61, 279-287.

Du, X., Ghosh, B. K., and Ulinski, P. (2005). Encoding and decoding target locations with waves in the turtle visual cortex. IEEE Trans. Biomed. Eng. 52, 566-577.

Ermentrout, B. (1986). "Losing amplitude and saving phase," in Nonlinear Oscillations in Biology and Chemistry, ed. H. G. Othmer (Proceedings of a meet- ing held at the University of Utah, May 9-11, 1985) (Berlin: Springer), 98.

Ermentrout, B. (1996). Type I membranes, phase resetting curves, and synchrony. Neural Comput. 8, 979-1001.

Ermentrout, G., and Kopell, N. (1990). Oscillator death in systems of coupled neural oscillators. SIAM J. Appl. Math. 50, 125-146.

Ermentrout, G., and Kopell, N. (1991). Multiple pulse interactions and averaging in systems of coupled neural oscillators. J. Math. Biol. 29, 195-217.

Ermentrout, G. B., and Kopell, N. (1986). Parabolic bursting in an excitable system coupled with a slow oscillation. SIAM J. Appl. Math. 46, 233-253.

Eusebio, A., and Brown, P. (2009). Synchronisation in the beta frequencyband - the bad boy of parkinsonism or an innocent bystander? Exp. Neurol. $217,1-3$.

Faisal,A., Selen, L., and Wolpert, D. (2008). Noise in the nervous system. Nat. Rev. Neurosci. 9, 292-303.

Fox, M., and Raichle, M. (2007). Spontaneous fluctuations in brain activity observed with functional magnetic resonance imaging. Nat. Rev. Neurosci. 8, 700-711.

Frank, T., Daffertshofer, A., and Beek, P. (2002). Impacts of statistical feedback 
on the flexibility-accuracy trade-off in biological systems. J. Biol. Phys. 28, 39-54.

Frank, T., Daffertshofer,A., Peper, C., Beek, P., and Haken, H. (2000). Towards a comprehensive theory of brain activity: coupled oscillator systems under external forces. Physica D 144, 62-86.

Freeman, W. J. (1975). Mass Action in the Nervous System: Examination of Neurophysiological Basis of Adoptive Behavior Through the EEG. New York: Academic Press.

Freyer, F., Aquino, K., Robinson, P., Ritter, P., and Breakspear, M. (2009). Bistability and non-Gaussian fluctuations in spontaneous cortical activity. J. Neurosci. 29, 8512.

Friston, K., and Dolan, R. (2009). Computational and dynamic models in neuroimaging. Neuroimage 52, 752-765.

Fujimoto, K., and Kaneko, K. (2003). How fast elements can affect slow dynamics. Physica D 180, 1-16.

Grinvald, A., Lieke, E. E., Frostig, R. D., and Hildesheim, R. (1994). Cortical pointspread function and long-range lateral interactions revealed by real-time optical imaging of macaque monkey primary visual cortex. J. Neurosci. 14 , 2545-2568.

Guckenheimer, J., and Holmes, P. (1990). Nonlinear oscillations, dynamical systems, and bifurcations of vector fields. Copernicus.

Haken, H. (1983). Synergetics, 3rd Edn. Berlin: Springer

Hansel, D., and Mato, G. (1993). Patterns of synchrony in a heterogeneous Hodgkin-Huxley neural network with weak coupling. Physica A 200, 662-669.

Hansel, D., Mato, G., and Meunier, C. (1993a). Clustering and slow switching in globally coupled phase oscillators. Phys. Rev. E 48, 3470.

Hansel, D., Mato, G., and Meunier, C. (1993b). Phase dynamics for weakly coupled Hodgkin-Huxley neurons. Europhys. Lett. 23, 367-372.

Hansel, D., Mato, G., and Meunier, C. (1995). Synchrony in excitatory neural networks. Neural Comput. 7, 307-337.

Hildebrand, E., Buice, M., and Chow, C. (2007). Kinetic theory of coupled oscillators. Phys. Rev. Lett. 98, 54101.

Honey, C., Kötter, R., Breakspear, M., and Sporns, O. (2007). Network structure of cerebral cortex shapes functional connectivity on multiple time scales. Proc. Natl. Acad. Sci. U.S.A. 104, 10240.

Hoppensteadt, F., and Izhikevich, E. (1998). Thalamo-cortical interactions modeled by weakly connected oscillators: could the brain use FM radio principles? Biosystems 48, 85-94.

Hoppensteadt, F. C., and Izhikevich, E. M. (1997). Weakly Connected Neural
Networks. New York, NY: Springer Verlag.

Houweling, S., Beek, P., and Daffertshofer, A. (2010). Spectral changes of interhemispheric crosstalk during movement instabilities. Cereb. Cortex 20, 2605-2613.

Jeong, S. O., Ko, T. W., and Moon, H. T. (2002). Time-delayed spatial patterns in a two-dimensional array of coupled oscillators. Phys. Rev. Lett. 89, 154104.

Kiebel, S., Daunizeau, J., and Friston, K. (2008). A hierarchy of time-scales and the brain. PLoS Comput. Biol. 4. 20, 2605-2613.

Kuramoto, Y.(1984).Chemical Oscillations, Waves, and Turbulence. Mineola, NY: Dover Publications.

Kuramoto, Y. (1997). Phase-and centermanifold reductions for large populations of coupled oscillators with application to non-locally coupled systems. Int. J. Bifurcat. Chaos 7 , 789-806.

Lam, Y. W., Cohen, L. B., Wachowiak, M., and Zochowski, M. R. (2000). Odors elicit three different oscillations in the turtle olfactory bulb. J. Neurosci. 20,749 .

Linkenkaer-Hansen, K., Nikouline, V., Palva, J., and Ilmoniemi, R. (2001). Long-range temporal correlations and scaling behavior in human brain oscillations. J. Neurosci. 21, 1370.

Ma, W., Beck, J., Latham, P., and Pouget, A. (2006). Bayesian inference with probabilistic population codes. Nat. Neurosci. 9, 1432-1438.

Mardia, K. (1972). Statistics of Directional Data. New York: Academic Press.

Miller, K. J., Sorensen, L. B., Ojemann, J. G., and den Nijs, M. (2009). Powerlaw scaling in the brain surface electric potential. PLoS Comput. Biol. 5, e1000609. doi: 10.1371/journal. pcbi.1000609.

Mirollo, R., and Strogatz, S. (2007). The spectrum of the partially locked state for the Kuramoto model. J. Nonlinear Sci. 17, 309-347.

Moss, F., Ward, L., and Sannita, W. (2004). Stochastic resonance and sensory information processing: a tutorial and review of application. Clin. Neurophysiol. 115, 267-281.

Orosz, G., Ashwin, P., and Townley, S. (2009). Learning of spatio-temporal codes in a coupled oscillator system. IEEE Trans. Neural Netw. 20, 1135-1147.

Penny, W., Litvak,V., Fuentemilla,L., Duzel, E., and Friston, K. (2009). Dynamic causal models for phase coupling. J. Neurosci. Methods 183, 19-30

Prechtl, J. C., Cohen, L. B., Pesaran, B., Mitra, P. P., and Kleinfeld, D. (1997). Visual stimuli induce waves of electrical activity in turtle cortex. Proc. Natl. Acad. Sci. U.S.A. 94, 7621-7626.
Risken, H. (1989). The Fokker-Planck Equation. Berlin: Springer.

Roland, P., Hanazawa, A., Undeman, C., Eriksson, D., Tompa, T., Nakamura, H., Valentiniene, S., and Ahmed, B. (2006). Cortical feedback depolarization waves: a mechanism of top-down influence on early visual areas. Proc. Natl. Acad. Sci. U.S.A. 103, 12586.

Rubino, D., Robbins, K., and Hatsopoulos, N. (2006). Propagating waves mediate information transfer in the motor cortex. Nat. Neurosci. 9, 1557-1549.

Sakaguchi, H. (1988). Cooperative phenomena in coupled oscillator systems under external fields. Prog. Theor. Phys. 79, 39-46.

Sakaguchi, H., Shinomoto, S., and Kuramoto, Y. (1988). Phase transition and their bifurcation analysis in large population of active rotators with mean-field coupling. Prog. Theor. Phys. 79, 600-607.

Schoner, G., and Kelso, J. (1988). Dynamic pattern generation in behavioral and neural systems. Science 239, 1513.

Schuster, H., and Wagner, P. (1990). A model for neuronal oscillations in the visual cortex. Biol. Cybern. 64, 77-82.

Sokolov, I., Klafter, J., and Blumen, A. (2002). Fractional kinetics. Phys. Today $55,48-54$.

Stam, C., and de Bruin, E. (2004). Scalefree dynamics of global functional connectivity in the human brain. Hum. Brain Mapp. 22, 97-109.

Stam, C., Nolte, G., and Daffertshofer, A. (2007). Phase lag index: assessment of functional connectivity from multi channel EEG and MEG with diminished bias from common sources. Hum. Brain Mapp. 28, 1178-1193.

Stratonovich, R. (1963). Topics in the Theory of Random Noise, Vols I, and II. New York: Gordon and Breach.

Strogatz, S. (2000). From Kuramoto to Crawford: exploring the onset of synchronization in populations of coupled oscillators. Physica D 143, 1-20.

Strogatz, S., and Mirollo, R. (1991). Stability of incoherence in a population of coupled oscillators. J. Stat. Phys. 63, 613-635.

Strogatz, S., Mirollo, R., and Matthews, P. (1992). Coupled nonlinear oscillators below the synchronization threshold: relaxation by generalized Landau damping. Phys. Rev. Lett. 68 2730-2733.

Tass, P. (1999). Phase Resetting in Medicine and Biology. Berlin: Springer.

Tass, P., Rosenblum, M., Weule, J., Kurths, J., Pikovsky, A., Volkmann, J., Schnitzler, A., and Freund, H. (1998). Detection of $\mathrm{n}$ : $\mathrm{m}$ phase locking from noisy data: application to magnetoencephalography. Phys. Rev. Lett. 81, 3291-3294.
Tsallis, C. (2006). Occupancy of phase space, extensivity of Sq, and q-generalized central limit theorem. Physica A 365, 7-16.

Tsallis, C., and Brigatti, E. (2004). Nonextensive statistical mechanics: a brief introduction. Continuum Mech. Thermodyn. 16, 223-235.

Varela, F., Lachaux, J., Rodriguez, E., and Martinerie, J. (2001). The Brainweb: phase synchronization and largescale integration. Nat. Rev. Neurosci. 2, 229-239.

Vreeswijk, C., Abbott, L., and Bard Ermentrout, G. (1994). When inhibition not excitation synchronizes neural firing. J. Comput. Neurosci. 1 , 313-321.

Wilson, H., and Cowan, J. (1973). A mathematical theory of the functional dynamics of cortical and thalamic nervous tissue. Biol. Cybern. 13, 55-80.

Winfree, A. (1967). Biological rhythms and the behavior of populations of coupled oscillators. J. Theor. Biol. 16, 15-42.

Wordsworth, J., and Ashwin, P. (2008). Spatiotemporal coding of inputs for a system of globally coupled phase oscillators. Phys. Rev. E 78, 66203.

Wu, J., Huang, X., and Zhang, C. (2008). Propagating waves of activity in the neocortex: what they are, what they do. Neuroscientist 14, 487-502.

Yeung, M. K. S., and Strogatz, S. H. (1999). Time delay in the Kuramoto model of coupled oscillators. Phys. Rev. Lett. 82, 648.

Zanette, D. (2000). Propagating structures in globally coupled systems with time delays. Phys. Rev. E 62, 3167-3172.

Zaslavsky, G. (2002). Chaos, fractional kinetics, and anomalous transport. Phys. Rep. 371, 461-580.

Conflict of Interest Statement: The authors declare that the research was conducted in the absence of any commercial or financial relationships that could be construed as a potential conflict of interest.

Received: 05 June 2010; accepted: 22 September 2010; published online: 11 November 2010.

Citation: Breakspear M, Heitmann S and Daffertshofer A (2010) Generative models of cortical oscillations: neurobiological implications of the Kuramoto model. Front. Hum. Neurosci. 4:190. doi: 10.3389/ fnhum. 2010.00190

Copyright $\odot 2010$ Breakspear, Heitmann and Daffertshofer. This is an open-access article subject to an exclusive license agreement between the authors and the Frontiers Research Foundation, which permits unrestricted use, distribution, and reproduction in any medium, provided the original authors and source are credited. 


\section{APPENDIX A}

\section{EMBEDDING TRANSMISSION-DELAYS IN A}

\section{SPATIAL KERNEL}

Consider a fully-connected Kuramoto model on a one-dimensional ring,

$\dot{\theta}_{n}=\omega_{n}+\frac{K}{N} \sum_{m=1}^{N} \sin \left(\theta_{m}-\theta_{n}-\alpha_{m n}\right)$,

that incorporates explicit phase-shifts $\alpha_{m n}$ that are proportional to the spatial distance $\left|x_{m}-x_{n}\right|$ between the oscillators ${ }^{7}$. A trivial steady-state wave solution exists when $\theta_{m}-\theta_{n}=\alpha_{m n}$ for all oscillator pairings, which corresponds to the phases of consecutive oscillators being distributed evenly on the unit circle. Such solutions have been observed by Zanette (2000). Here, we derive an equivalent model in which the explicit phase-shifts $\alpha_{m n}$ are embedded in a spatial kernel as coupling terms with no explicit delay terms.

Application of the trigonometric identity $\sin (x-y)=\sin x \cos$ $y-\cos x \sin y$ to equation (24) yields the equivalent form,

$$
\begin{aligned}
\dot{\theta}_{n} & =\omega_{n}+\frac{K}{N} \sum_{m=1}^{N} \cos \alpha_{m n} \sin \left(\theta_{m}-\theta_{n}\right) \\
& +\frac{K}{N} \sum_{m=1}^{N} \sin \alpha_{m n} \cos \left(\theta_{m}-\theta_{n}\right) .
\end{aligned}
$$

\footnotetext{
${ }^{7}$ Such phase-shifts are akin to distance-dependent transmission delays.
}

We argue that, when this system is close to a wave solution, the phases of the oscillators are aligned symmetrically on the unit circle so that $\left(\theta_{m}-\theta_{m-k}\right) \approx\left(\theta_{m}-\theta_{m+k}\right)$ for $k=1, \ldots, N / 2$. We also assume that all oscillators in the ring are equally spaced so that $\alpha_{m(m+k)}=-\alpha_{m(m-k)}$. Consequently, the final term in (25) may be re-expressed as

$\frac{K}{N} \sum_{m=1}^{N} \sin \alpha_{m n} \cos \left(\theta_{m}-\theta_{n}\right)$

$\approx \frac{K}{N} \sum_{k=1}^{N / 2} \sin \alpha_{m(m-k)} \cos \left(\theta_{m}-\theta_{m-k}\right)+\frac{K}{N} \sum_{k=1}^{N / 2} \sin \alpha_{m(m+k)} \cos \left(\theta_{m}-\theta_{m+k}\right)$

$\approx \frac{K}{N} \sum_{k=1}^{N / 2} \sin \alpha_{m(m-k)} \cos \left(\theta_{m}-\theta_{m-k}\right)-\frac{K}{N} \sum_{k=1}^{N / 2} \sin \alpha_{m(m-k)} \cos \left(\theta_{m}-\theta_{m-k}\right)$

$\approx 0$,

thus allowing Eq. 25 to be approximated by

$\dot{\theta}_{n}=\omega_{n}+\frac{K}{N} \sum_{m=1}^{N} W(m, n) \sin \left(\theta_{m}-\theta_{n}\right)$,

where the coupling coefficients $W_{(m, n)}=\cos \left(\alpha_{m n}\right)$ constitute a spatial kernel that varies periodically with distance and explicit phase-shift terms have been eliminated from the sinusoidal interaction term. 